\title{
Marginal pinning of vortices at high temperature
}

\author{
M. Müller ${ }^{1,2}$, D.A. Gorokhov ${ }^{1,3}$, and G. Blatter ${ }^{1}$ \\ ${ }^{1}$ Theoretische Physik, ETH-Hönggerberg, CH-8093 Zürich, Switzerland \\ ${ }^{2}$ LPTMS, Université Paris Sud, Bât. 100, F-91405 Orsay, France \\ ${ }^{3}$ Department of Physics, Harvard University, Cambridge, Massachusetts 02138
}

(October 31, 2018)

\begin{abstract}
We analyze the competition between thermal fluctuations and pinning of vortices in bulk type II superconductors subject to point-like disorder and derive an expression for the temperature dependence of the pinning length $L_{c}(T)$ which separates different types of single vortex wandering. Given a disorder potential with a basic scale $\xi$ and a correlator $K_{0}(u) \sim K_{0}(u / \xi)^{-\beta} \ln ^{\alpha}(u / \xi)$ we determine the dependence of $L_{c}(T)$ on the correlator range: correlators with $\beta>2$ (short-range) and $\beta<2$ (long-range) lead to the known results $L_{c}(T) \sim L_{c}(0) \exp \left[C T^{3}\right]$ and $L_{c}(T) \sim L_{c}(0)(C T)^{(4+\beta) /(2-\beta)}$, respectively. Using functional renormalization group we show that for $\beta=2$ the result takes the interpolating form $L_{c}(T) \sim L_{c}(0) \exp \left[C T^{3 /(2+\alpha)}\right]$. Pinning of vortices in bulk type II superconductors involves a long-range correlator with $\beta=2, \alpha=1$ on intermediate scales $\xi<u<\lambda$, with $\xi$ and $\lambda$ the coherence length and London penetration depth, hence $L_{c}(T) \sim L_{c}(0) \exp [C T]$; at large distances $L_{c}(T)$ crosses over to the usual short-range behavior.

PACS numbers: 05.20.-y, 64.60.Cn, 74.60.Ge
\end{abstract}

Many properties of type II superconductors derive from the interaction between vortices and pinning centers1.2. Impurities are particularly important in situations where a transport current is applied as they provide the necessary pinning force compensating the Lorentz force acting on vortices 3 and thus enable a dissipationfree current flow. In this note we address specific aspects of the high temperature pinning behavior of vortices subject to weak point-like disorder. The results are of particular relevance for the copper-oxide high $T_{c}$ superconductors which can be operated at high temperatures and where such a pinning landscape naturally derives from oxygen vacancies.

A single flux line in addisorder potential is described by the partition function 1

$$
\begin{aligned}
& Z(\mathbf{u}, L)=\int_{(\mathbf{0}, 0)}^{(\mathbf{u}, L)} \mathcal{D}\left[\mathbf{u}^{\prime}(z)\right] \\
& \exp \left\{-\frac{1}{T} \int_{0}^{L} d z\left[\frac{c}{2}\left(\frac{d \mathbf{u}^{\prime}}{d z}\right)^{2}+V\left(\mathbf{u}^{\prime}(z), z\right]\right)\right\}
\end{aligned}
$$

with $(c / 2)\left(\partial_{z} \mathbf{u}\right)^{2}$ the elastic energy and $V(\mathbf{u}, z)$ the disorder potential which might take negative values. The disorder is chosen to be a Gaussian random variable with zero mean and a correlator

$$
\langle V(\mathbf{u}, z) V(\mathbf{0}, 0)\rangle=K_{0}(\mathbf{u}) \delta(z),
$$

where $\langle\ldots\rangle$ denotes the average over disorder realizations. The correlator $K_{0}(\mathbf{u})$ decays on a length $\xi$; in most applications the function $K_{0}(\mathbf{u})$ is assumed to be rapidly decaying, and at sufficiently high temperatures the physical behavior is determined by the integral $\Delta \equiv \int d^{n} u K_{0}(\mathbf{u})$ alone. In this paper we draw attention to the situation in disordered type II superconductors where the correlator $K_{0}(\mathbf{u})$ describing the potential landscape of vortices is long-ranged,

$$
K_{0}(\mathbf{u}) \sim K_{0} \frac{\xi^{2}}{u^{2}} \ln \frac{u}{\xi},
$$

in the intermediate asymptotic regime $\xi<u<\lambda$ (here, $\xi$ and $\lambda$ denote the coherence length and the London penetration depth of the superconductor). The long-range tail (3) renders the correlator non-integrable and modifies the pinning characteristics as compared to a short-range correlated disorder landscape. This effect is particularly pronounced for vortices in bulk high- $T_{c}$ superconductors where the disorder becomes marginal at high temperatures and the ratio $\lambda / \xi$ is large (below we describe the situation in an isotropic material; effects of anisptropy can be accounted for within the scaling approachl).

The following discussion is not restricted to single vortex pinning; rather we consider the latter as a specific realizatign of the directed elastic string (or polymer-) problem which describes such diverse physical systems as domain walls in magnetic films 6 wetting (in the plane) 8 , vorticest or random polymers 9 . The numerous non-trivial features that these systems have in common derive from an intricate interplay of elasticity, disorder and thermal fluctuations. While the elastic forces tend to stretch the string, the disorder potential favors configurations deviating from a straight line in order to take advantage of the potential valleys. Within the weak collective pinning scenario 10 the elasticity dominates on scales smaller than the cross-over scale $L_{c}$ and forces the string to stay in the same valley, whereas on larger scales the string effectively divides up into segments of size $L_{c}$ which adjust independently to the disorder landscape. Increasing the disorder strength decreases $L_{c}$, while thermal fluctuations tend to smooth the disorder landscape implying an increase of $L_{c}(T)$ with temperature. Also, the typical 
barriers separating adjacent valleys are reduced by thermal fluctuations leading to a peculiar form of the creeptype dynamics under a small external force 11 . Below we first discuss some general properties of vortex pinning in disordered type II superconductors and derive the asymptotic form (3) of the correlator. Second, we calculate the pinning length $L_{c}(T)$ using the functional renormalization group (FRG) approach 12 .

The behavior of random directed polymers strongly depends on the number $n$ of transverse motional degrees of freedom. E.g., the (low temperature) roughness as characterized by the wandering exponent $\zeta_{n}=$ $\lim _{L \rightarrow \infty} \partial \ln \left\langle u^{2}(L)\right\rangle / \partial \ln L^{2}$ decreases with $n$ (here, $L$ denotes the length of the polymer segment and $\mathbf{u}(L)$ is the relative transverse displacement of its end points). Upon increasing the temperature a phase transition (roughening transition) is known to occur for $n \geq 3$, see Ref. 13, the high temperature phase being dominated by thermal fluctuations on all length scales with a thermal roughness exponent $\zeta_{\text {th }}=1 / 2$. On the other hand, for $n=1,2$ the large scale behavior remains dominated by disorder with the same exponent $\zeta_{n}$ as at low temperatures, however, going beyond the so-called depinning temperature $T_{\mathrm{dp}}$, the crossover scale $L_{c}(T)$ increases rapidly with temperature. In the physically important case $n=2$ the crossover length $L_{c}(T)$ grows exponentially, $L_{c}(T) \sim L_{c}(0) \exp \left[C\left(T / T_{\mathrm{dp}}\right)^{\nu}\right]$, reflecting the fact that $n=2$ is the lower critical dimension of the roughening transition. While $\nu=3$ is a well established result descriping the situation for a short-range disorder potential14, the exponent is modified by the long-range tail of the potential correlator as it appears in the vortex problem: we will show below that $\nu=1$ (a similar effect is found in the context of individual vortices pinned onto columnar tracks2). We emphasize that the asymptotic behavior (3) does not influence the roughness of the polymer on large scales, that is, the value of the wandering exponent $\zeta_{n}$ remains unchanged. While for $n=2$ the non-integrability of the function $K_{0}(u)$ with an asymptotic decay slower than $1 /\left[u^{2} \ln (u)\right]$, is sufficient to change the value of the exponent $\nu$, the criterion on the asymptotics $K_{0}(u) \sim K_{0}(\xi / u)^{\beta}$ to change the value of $\zeta_{2}$ has been argued 15 , 16 to be $\beta<3 / \zeta_{2, \text { sr }}-4$. Inserting the numerically known value $\zeta_{2, \mathrm{sr}} \approx 5 / 8$ for the short-range wandering exponent, we see that the $\zeta_{2}=\zeta_{2, \mathrm{sr}}$ is unaffected by the weak non-integrability $(\beta=2)$ in the vortex pinning problem.

We briefly derive the form of the long-range correlations occurring in the problem of single vortex pinning in type II superconductors 2 . The GinzburgLandau equation for the macroscopic wave function $\Psi=$ $\sqrt{\rho(R)} \exp (i \varphi)$ takes the form

$$
\xi^{2}\left(\nabla+\frac{2 \pi i}{\Phi_{0}} \mathbf{A}\right)^{2} \Psi+\left(1-|\Psi|^{2}\right) \Psi=0,
$$

where $\mathbf{A}$ denotes the vector potential, $\Phi_{0}=h c / 2 e$ is the flux unit, and we have normalized $|\Psi|$ to unity in the asymptotic regime. We concentrate on the vortex solution where the phase turn in $\varphi$ by $2 \pi$ drives a circular vortex current. On scales $R<\lambda$ we can ignore transverse screening and using $(\nabla \varphi)^{2}=1 / R^{2}$, the real part of (4) simplifies to $\left(-\xi^{2} / R^{2}+1-|\Psi|^{2}\right) \Psi=0$, hence

$$
|\Psi|^{2}(\xi \ll R<\lambda) \approx 1-\xi^{2} / R^{2}
$$

the circular vortex current produces an order parameter suppression decaying only slowly at small distances $R<\lambda$ (transverse screening quenches the current flow beyond the screening length $\lambda$ and the suppression of the order parameter is exponentially small) The same result follows from a variational Ansatz $1718, \Psi=R /\left(R^{2}+\right.$ $\left.2 \xi^{2}\right)^{1 / 2} \exp (i \varphi)$. In high temperature superconductors $\lambda$ is much larger than $\xi$ and the slow decay of the order parameter extends over a wide region.

To fix ideas, we consider disprder in the critical temperature (so called $\delta T_{c}$-disorder 4 ) described through spatial variations $\delta \alpha(\mathbf{r})$ in the Ginzburg-Landau parameter $\alpha$ with correlations $\left\langle\delta \alpha(\mathbf{r}) \delta \alpha\left(\mathbf{r}^{\prime}\right)\right\rangle=\gamma \delta^{3}(\mathbf{r}-\mathbf{r})$. The pinning energy of a vortex aligned along the $z$-axis and with coordinates $(\mathbf{u}(z), z)$ is given by

$$
E_{\text {pin }}(\mathbf{u}, z)=\int d^{2} R p(|\mathbf{u}-\mathbf{R}|) \delta \alpha(\mathbf{R}, z),
$$

with the vortex form factor $p(R)=1-|\Psi(R)|^{2}=$ $2 \xi^{2} /\left(R^{2}+2 \xi^{2}\right)$. Within the range $\xi \ll u<\lambda$ the pinning energy correlator assumes the form

$$
\begin{aligned}
& K_{0}(u) \equiv\left\langle E_{\operatorname{pin}}(\mathbf{u}, z) E_{\operatorname{pin}}(\mathbf{0}, 0)\right\rangle \\
& =\gamma \delta(z) \int d^{2} R p(|\mathbf{u}-\mathbf{R}|) p(R) \sim K_{0} \delta(z) \frac{\xi^{2}}{u^{2}} \ln \frac{u}{\xi}
\end{aligned}
$$

with $K_{0}=2 \pi\left|\Psi_{0}\right|^{4} \gamma \xi^{2}$ (we have assumed $\left|\Psi_{0}\right|=1$ in the derivation above). The integral over this correlator diverges logarithmically and is cut off only at the large scale $\lambda$. This long-range feature will have an important effect on the cross-over scale $L_{c}(T)$ at high temperatures which we are now going to calculate.

We analyze the system described by the partition function (11) with the help of the functional renormalization group (FRG) approach 12 . Applying momentum shell renormalization to the replicated Hamiltonian leads to the following system of one loop equations (see Ref. 19) for the renormalized correlator $K_{l}$ and temperature $T_{l}$,

$$
\begin{aligned}
\partial_{l} K_{l}(|\mathbf{u}|) & =[3-(4+n) \zeta] K_{l}(|\mathbf{u}|) \\
& +\zeta \nabla \cdot\left(\mathbf{u} K_{l}(|\mathbf{u}|)\right)+\tilde{T}_{l} K_{l}^{\mu \mu}(|\mathbf{u}|) \\
& +I\left[K_{l}^{\mu \nu}(|\mathbf{u}|) K_{l}^{\mu \nu}(|\mathbf{u}|) / 2-K_{l}^{\mu \nu}(|\mathbf{u}|) K_{l}^{\mu \nu}(0)\right] \\
\partial_{l} \tilde{T}_{l} & =(1-2 \zeta) \tilde{T}_{l}
\end{aligned}
$$

where $I=1 /\left(\pi c^{2} \Lambda^{3}\right), \tilde{T}_{l}=T_{l} /(\pi c \Lambda)$, and $\Lambda^{-1}$ denotes the short-scale cut-off of the theory. In (8) we limited ourselves to the two replica correlator $K_{l}$, neglecting higher replica terms generated at high temperatures during momentum shell integration, see Ref. 19: an analysis including these terms reveals that their feedback to the flow of 
$K_{l}$ is of the same order as the non-linear terms in (8) and hence the qualitative results obtained below remain valid.

At zero temperature the fourth derivative of the correlator $K_{l}^{(4)}(0)$ diverges at the finite scale $l_{c}(T=0) \approx$ $\ln \left\{9 /\left[(n+8) I K_{0}^{(4)}(0)\right]\right\}$, signalling the emergence of a nonanalyticity in the correlator at the origin and indicating that perturbation theory in the disorder breaks down beyond this scale. Still, the FRG-flow remains well-defined, and for the case of short-range disorder the correlator can be shown to flow rapidly towards a non-analytic disorderdominated fixed point beyond $l_{c}(0)$. The crossover scale? $L_{c}(0)=\Lambda^{-1} e^{l_{c}(0)} \approx\left(c^{2} / K_{0}^{(4)}(0)\right)^{1 / 3}$ is naturally interpreted as the typical length of independently pinned vortex segments within the weak collective pinning theory; the same result is obtained from a simple scaling argument equating the elastic and disorder energies.

At finite temperatures the correlator remains analytic due to the thermal smearing introduced via the term $\tilde{T} K_{l}^{\mu \mu}$. However, we can still identify the crossover scale $l_{c}(\tilde{T})$ as the value of $l$ where the non-linear terms in Eq. (8) become of the same order as the linear terms, indicating that the disorder cannot any longer be dealt with perturbatively; beyond this scale $K_{l}$ is again driven towards the $(T=0)$ strong coupling fixed point.

At small scales, the non-linear terms in the flow equation (8) for the correlator $K_{l}$ can be neglected. Furthermore, since we want to study high temperatures, we choose the roughness exponent to take the thermal value $\zeta=\zeta_{\text {th }}=1 / 2$ which is convenient since the physics appears more transparently in the sequel, in particular, the temperature does not renormalize when $\zeta=\zeta_{\text {th }}$ and $\tilde{T}_{l}=\tilde{T}$ (however, note that from a mathematical point of view the physical results below are independent of this particular choice of $\zeta$ ). The linearized flow equation can be solved explicitly 11 with the result

$$
\begin{aligned}
K_{l}(u)= & e^{(1-n / 2) l}\left[\frac{1}{4 \pi \tilde{T}\left(1-e^{-l}\right)}\right]^{n / 2} \\
& \times \int \mathrm{d}^{n} u^{\prime} \exp \left[-\frac{\left(\mathbf{u}-\mathbf{u}^{\prime} e^{-l / 2}\right)^{2}}{4 \tilde{T}\left(1-e^{-l}\right)}\right] K_{0}\left(u^{\prime}\right) .
\end{aligned}
$$

Let us analyze the situation for short- and long-range correlated disorder separately. If the disorder correlator is short-ranged, the total weight $\Delta \equiv \int d^{n} u^{\prime} K_{0}\left(u^{\prime}\right)$ is finite and the expression (10) can be simplified at high temperatures $\tilde{T} \gg \tilde{T}_{\mathrm{dp}} \equiv \xi^{2} e^{-l_{c}(0)}$,

$$
K_{l}(u)=e^{(1-n / 2) l} \Delta \tilde{T}^{-n / 2} \exp \left[-u^{2} / 4 \tilde{T}\right],
$$

for $\max \left[1, \ln \left(\xi^{2} / \tilde{T}\right)\right] \ll l<l_{c}(0)$. Note that for $n=1$ the correlator grows exponentially, reflecting the relevance of the disorder, whereas in the marginal case $n=2$ the correlator flows to a fixed point. In this case we can interpret the linearized flow equation as the Fokker-Planck equation for the probability distribution $K_{l}(u)$ of a particle in a harmonic potential $V(u)=u^{2} / 4$, where $l$ plays the role of time and $\tilde{T}$ is the temperature. The above fixed point corresponds to the stationary Gibbs-Boltzmann distribution which is rapidly approached at high temperatures.

In the case of long-range correlations the integration kernel in Eq. (10) cuts the non-integrable tails of the correlator at $u^{\prime} \sim\left(e^{l} \tilde{T}\right)^{1 / 2}$ and we may estimate the renormalized correlator at the origin as

$$
K_{l}(0) \sim e^{(1-n / 2) l}(4 \tilde{T})^{-n / 2} \int_{0}^{\left(e^{l} \tilde{T}\right)^{1 / 2}} K_{0}(u) u^{n-1} d u .
$$

In the case $n=2$ this expression can be understood in terms of the diffusive motion of "particles" that are initially distributed with a density proportional to $K_{0}(u)$. Their total number is infinite due to the non-integrable tails of $K_{0}(u)$ (see Eq. (7) ), and a growing number of "particles" from further and further away will eventually accumulate at the origin, continuously increasing $K_{l}(0)$ (see Fig. 1). Thermal equilibrium is never reached in this situation.

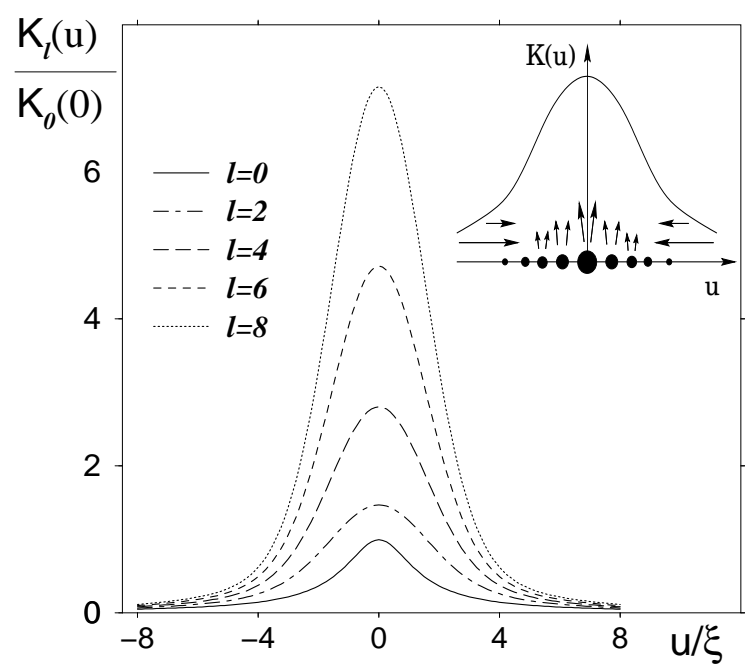

FIG. 1. The high temperature $\left(\tilde{T}=\xi^{2}\right)$ diffusive flow of the correlator $K_{l}(u)$ evolving under the linearized FRG for $n=2$. We choose a bare correlator $K_{0}(u)=\left\{1+\ln \left[1+(u / \xi)^{2}\right] / 2\right\} /\left[1+(u / \xi)^{2}\right]$ with non-integrable logarithmic tails as in the vortex problem. After a crossover around $l=1$ the correlator $K_{l}(0)$ increases quadratically with $l$ as expected from Eq. (13). The inset schematically illustrates the interplay of the diffusive inflow of "particles" (horizontal arrows) and the "particle" sources originating from the quadratic one-loop terms (solid dots). In the short-range case the first mechanism is absent and $K_{l}$ grows due to the non-linear terms only, while in the strongly long-range case $(\beta<2)$, the non-linear terms can be neglected and $K_{l}$ grows due to the inflow from the tails. The vortex pinning problem is intermediate with a crossover at a scale $l_{\mathrm{cr}}(\tilde{T})<l_{c}(\tilde{T})$ from diffusion driven growth to source driven growth.

In the following we first discuss the situation where the linearized flow gives rise to an exponential growth of the renormalized correlator at the origin. This is the case 
for $n=1$, or for $n=2$ if the initial correlator decays asymptotically as $K_{0}(u) \sim K_{0}(\xi / u)^{\beta}$ with an exponent $\beta<2$. Under these conditions, the contribution of the non-linear terms to the growth of $K_{l}$ can be neglected, and the crossover scale $l_{c}(\tilde{T})$ is found from comparing linear and quadratic terms in the flow equation, i.e.,

$$
I K_{l_{c}(\tilde{T})}^{\prime \prime}(0) \sim \tilde{T} .
$$

The second derivative of $K_{l}$ can be estimated from Eq. 10), $K_{l}^{\prime \prime}(0) \approx K_{l}(0) / \tilde{T}$. Using expression (12) in the above crossover condition we find

$$
L_{c}\left(T>T_{\mathrm{dp}}\right) \sim L_{c}(0)\left(\frac{T}{T_{\mathrm{dp}}}\right)^{(4+\beta) /(2-\beta)}
$$

with the depinning temperature

$$
T_{\mathrm{dp}} \approx c \frac{\xi^{2}}{L_{c}(0)} .
$$

A short-range correlator with $n=1$ requires $\beta=1$ and one recover 20 the well-known dependence $L_{c}(T) \sim$ $L_{c}(0)\left(T / T_{\mathrm{dp}}\right)^{5}$. The result (14) can also be derived from simple scaling arguments, c.f., Refs. 2,11.

The marginal case $n=2$ is more subtle if the correlator is short-ranged or only weakly non-integrable with an asymptotic behavior $K_{0}(u) \sim K_{0}(\xi / u)^{2} \ln ^{\alpha}(u / \xi)$, where $\alpha>-1$. In the short-range case the "Boltzmannian" fixed point (11) is unstable only due to the quadratic loop corrections in the FRG. They can be considered as sources of "particles" in the picture introduced above (see Fig. 1). Well below the crossover $l_{c}(\tilde{T})$, these new particles will quickly thermalize, and the shape of the distribution will remain Boltzmannian, whereas its total weight will grow. Qualitatively, we obtain $l_{c}(\tilde{T})$ as follows: From Eqs. (10) and (11) $K_{l}$ is seen to vary on a typical scale $\tilde{T}^{1 / 2}$ thus the quadratic terms on the right-hand side of Eq. (8) can be estimated by $A I\left[K_{l}(0) / \tilde{T}\right]^{2}$ for $u \leq \tilde{T}^{1 / 2}$ with $A$ a numerical prefactor. The amplitude of the correlator is determined from the flow equation

$$
\partial_{l} K_{l}(0) \simeq A \frac{I}{\tilde{T}^{2}} K_{l}^{2}(0) .
$$

Integrating over the interval $\max \left[1, \ln \left(\xi^{2} / \tilde{T}\right)\right]<l<l_{c}(\tilde{T})$ and using the crossover condition (13) as well as the initial shape Eq. (11) one recovers the well-known result $L_{c}(T) \sim L_{c}(0) \exp \left[C\left(T / T_{\mathrm{dp}}\right)^{3}\right]$. Here and in the following we make use of the fact that the details of the initial condition $K_{0}(u)$ are quickly washed out, giving way to a correlator of Boltzmann shape (11); the latter serves as our new initial condition. For the short-range case an exact flow equation for the total weight can be written down under the assumption that the Boltzmannian shape of the correlator is preserved. This allows to determine the constant $C=32 / \pi$ exactly, see Ref. 19 .

Let us then discuss the case of a weakly long-range correlator with a tail $K_{0}(u) \sim K_{0}(\xi / u)^{2} \ln ^{\alpha}(u / \xi)$. Note that now the total weight of the correlator diverges and the linearized flow does not approach a fixed point. However, the flow of the central part of the correlator is still governed by the linear terms in the flow equation (8) which force the correlator to maintain an almost Boltzmannian shape of width $(4 \tilde{T})^{1 / 2}$. We will therefore assume that the flow in the region $u \leq \tilde{T}^{1 / 2}$ is captured by the growth of the amplitude $K_{l}(0)$, and that second derivatives may be replaced up to numerical factors by $1 / \tilde{T}$. In the first stage of the FRG-flow, the growth of $K_{l}(0)$ is dominated by the diffusive flow of particles to the origin as described by (12). However, at an intermediate scale $l_{\mathrm{cr}}(\tilde{T})<l_{c}(\tilde{T})$ the quadratic source terms become non-negligible and finally dominate, the subsequent flow being analogous to the short-range case. In order to determine the scale $l_{\mathrm{cr}}(\tilde{T})$ we compare the rate of growth due to diffusion, Eq. (12), with the magnitude of the quadratic terms in Eq. (8),

$$
\partial_{l} K_{l}^{\operatorname{diff}}(0) \stackrel{(12)}{\sim} \frac{\left[K_{0}(u) u^{2}\right]_{u=\left(e^{l_{\mathrm{cr}} \tilde{T}}\right)^{1 / 2}}}{8 \tilde{T}} \sim I\left(\frac{K_{l_{\mathrm{cr}}}(0)}{\tilde{T}}\right)^{2} .
$$

Assuming the bare correlator to decay asymptotically as $K_{0}(u) \sim K_{0}(\xi / u)^{2} \ln ^{\alpha}(u / \xi)$ and evaluating the righthand side of (17) with the help of Eq. (12) we find that

$$
l_{\mathrm{cr}}(\tilde{T}) \sim\left(\frac{\tilde{T}}{\tilde{T}_{\mathrm{dp}}}\right)^{3 /(2+\alpha)} .
$$

Note that we have to require that $\alpha>-1$, otherwise the inflow of "particles " will saturate already at small scales and the crossover condition (17) cannot be applied.

The further flow will be dominated by the non-linear terms while the linear terms still make sure that the overall shape of $K_{l}$ resembles a Boltzmannian of width $(4 \tilde{T})^{1 / 2}$. Only beyond the crossover scale $l_{c}(\tilde{T})$ will the non-linear terms be large enough to drive the correlator to a disorder dominated fixed point. As in the shortrange case, $K_{l}(0)$ satisfies the approximate equation (16) in the region $l_{\mathrm{cr}}(\tilde{T})<l<l_{c}(\tilde{T})$. Integrating the latter beyond $l_{\mathrm{cr}}(\tilde{T})$ and using the condition (13) we find

$$
l_{c}(\tilde{T})-l_{\mathrm{cr}}(\tilde{T}) \sim\left(\frac{\tilde{T}}{\tilde{T}_{\mathrm{dp}}}\right)^{3 /(2+\alpha)},
$$

and, recalling $l=\ln (\Lambda L)$,

$$
L_{c}(T) \sim L_{c}(0) \exp \left[C\left(\frac{T}{T_{\mathrm{dp}}}\right)^{3 /(2+\alpha)}\right]
$$

with an unknown numerical factor $C$; its determination as well as the precise value of the prefactor is beyond the accuracy of the present analysis. The short-range result with an exponent proportional to $T^{3}$ is recovered for $\alpha=-1$ which corresponds to the limiting case of an integrable correlator $(\alpha<-1)$. Eq. (20) shows that at 
a given (high) temperature the crossover scale $L_{c}(T)$ is the smaller the larger is the range of the potential correlations, turning finally to a power law (14) for strongly long-range correlators with $\beta<2$. Note that while both the long- and short-range results derive from cutting the flow through the condition (13), the discussion of the intermediate range correlator with $\beta=2$ and $\alpha>-1$ involves the additional crossover length $l_{\text {cr }}(\tilde{T})$ which derives from the condition (17). The latter condition then determines the exponent $3 /(2+\alpha)$ providing the smooth interpolation between the long- and short-range results.

Single vortex pinning is described by $\alpha=1$ which leads to an exponent $\propto T$. However, in the above derivation we have not taken into account that the logarithmic tails (7) only extend up to $\lambda$. The result (20) only applies if at $l_{\text {cr }}(\tilde{T})$ the integral $(10)$ is still cut by the Boltzmann kernel such that the estimate (12) remains valid. We may obtain an upper bound $\tilde{T}_{u}$ on $\tilde{T}$ by requiring that $\left(e^{l_{\mathrm{cr}}\left(\tilde{T}_{u}\right)} \tilde{T}_{u}\right)^{1 / 2}=\lambda$ in the crossover $(17)$. At temperatures higher than $\tilde{T}_{u}$ a crossover to the short-range case will take place. Finally, the range of validity of 20 is found to be

$$
T_{\mathrm{dp}}<T<T_{u} \sim T_{\mathrm{dp}} \ln ^{\frac{2+\alpha}{3}}(\kappa)=T_{\mathrm{dp}} \ln (\kappa) .
$$

Apart from the restriction on the temperature the magnetic field $B$ has to be weak such that effects due to vortex-vortex interactions can be ignored; the corresponding condition $L_{c}(T)^{2}<\Phi_{0} / B$ derives from comparing tilt and shear energiest.

In conclusion, we have determined the temperature dependence of the collective pinning length $L_{c}(T)$ for a directed elastic string subject to a long-range correlated disorder potential. In the physically important case of single vortex pinning in three-dimensional bulk material $L_{c}(T)$ exhibits an exponential sensitivity to temperature: in the intermediate temperature range $T_{\mathrm{dp}}<T<$ $(\ln \kappa) T_{\mathrm{dp}}$ the logarithmic tails of the correlator produce a simple exponential law $L_{c}(T) \sim L_{c}(0) \exp \left[C\left(T / T_{\mathrm{dp}}\right)\right]$, while for $T>(\ln \kappa) T_{\mathrm{dp}}$ the usual short-range result $L_{c}(T) \sim L_{c}(0) \exp \left[C\left(T / T_{\mathrm{dp}}\right)^{3}\right]$ holds.
${ }^{1}$ T. Nattermann and S. Scheidl, Adv. Phys. 49, 607 (2000).

${ }^{2}$ G. Blatter, M.V. Feigel'man, V.B. Geshkenbein, A.I. Larkin, and V.M. Vinokur, Rev. Mod. Phys. 66, 1125 (1994).

${ }^{3}$ M. Tinkham, Introduction to Superconductivity (McGrawHill, New York, 1996).

${ }^{4}$ T. Halpin-Healy and Y.-C. Zhang, Phys. Rep. 254, 215 (1995).

${ }^{5}$ G. Blatter, V.B. Geshkenbein, and A.I. Larkin, Phys. Rev. Lett. 68, 875 (1992).

${ }^{6}$ S. Lemerle, J. Ferré, C. Chappert, V. Mathet, T. Giamarchi and P. Le Doussal, Phys. Rev. Lett. 80, 849 (1998).

${ }^{7}$ G. Forgacs, R. Lipowsky, and Th.M. Nieuwenhuizen, in Phase Transitions and Critical Phenomena, Vol. 14, eds. C. Domb and J. Lebowitz (Academic Press, London, 1991).

${ }^{8}$ D. Wilkinson and J. F. Willemsen, J. Phys. A 16, 3365, (1983)

${ }^{9}$ M. Kardar and Y.-C. Zhang, Phys. Rev. Lett. 58, 2087 (1987).

10 A.I. Larkin, Sov. Phys. JETP 31784 (1970), A.I. Larkin and Y.N. Ovchinnikov, J. Low Temp. Phys. 34409 (1979).

${ }^{11}$ M. Müller, D. A. Gorokhov and G. Blatter, condmat/0012315 (2000).

${ }^{12}$ D.S. Fisher, Phys. Rev. Lett. 56, 1964 (1986).

13 J.Z. Imbrie and T. Spencer, J. Stat. Phys. 52, 609 (1988).

${ }^{14}$ M.V. Feigel'man and V.M. Vinokur, Phys. Rev. B 41, 8986 (1990).

15 T. Halpin-Healy, Phys. Rev. Lett. 62, 442 (1989).

${ }^{16}$ L. Balents and D. Fisher, Phys. Rev. B 48, 5949 (1993).

17 A. Schmid, Phys. Kondens. Materie 5, 302 (1966).

18 J.R. Clem, J. Low Temp. Phys. 18427 (1975).

19 D.A. Gorokhov and G. Blatter, Phys. Rev. B 62, 14032 (2000).

${ }^{20}$ L.B. Ioffe and V.M. Vinokur, J. Phys. C 20, 5149 (1987). 